\title{
NUCLEAR $C^{*}$-ALGEBRAS HAVE AMENABLE UNITARY GROUPS
}

\author{
ALAN L. T. PATERSON
}

(Communicated by Paul S. Muhly)

\begin{abstract}
Let $A$ be a unital $C^{*}$-algebra with unitary group $G$. Give $G$ the relative (Banach space) weak topology. Then $G$ is a topological group, and we show that $A$ is nuclear if and only if there exists a left invariant mean on the space of right uniformly continuous, bounded, complex-valued functions on $G$.
\end{abstract}

Nuclear $C^{*}$-algebras and injective von Neumann algebras are of fundamental importance in operator algebra theory. The two classes of algebras are related through the remarkable result (of Choi-Effros and Connes): $a C^{*}$-algebra $A$ is nuclear if and only if $A^{* *}$ is an injective von Neumann algebra.

From the work of Haagerup [3], nuclearity is the same as amenability (in the Banach algebra sense) for $C^{*}$-algebras. Further, from the deep work of Connes and others, it is known that injectivity, Property $\mathrm{P}$, hyperfiniteness and amenability are all equivalent for a von Neumann algebra $M$.

The relationship between injectivity and classical amenability for topological groups is established in a result of de la Harpe [4] discussed below. We note here that Haagerup in [3] proves that the injectivity of $M$ is equivalent to the existence of a left invariant mean on a certain space of functions on the isometry semigroup of $M$. The author plans to discuss the relationship between the invariant mean results of Haagerup and de la Harpe in a future paper.

We recall some notions from topological group theory. A fundamental system of entourages for the right uniformity on a topological group $G$ is given by sets of the form

$$
\left\{(x, y) \in G \times G: y x^{-1} \in V\right\},
$$

where $V$ is a neighborhood of the identity $e$ in $G$. Let $\operatorname{RUC}(G)$ be the space of right uniformly continuous bounded functions $f: G \rightarrow \mathbf{C}$. It is well known and easy to show that if $f: G \rightarrow \mathbf{C}$ is bounded, then $f \in \operatorname{RUC}(G)$ if and only if the map $x \rightarrow f x$ is norm continuous from $G$ to $l_{\infty}(G)$, where $f x(y)=f(x y)$ $(y \in G)$. The space $\operatorname{RUC}(G)$ is a unital $C^{*}$-subalgebra of $l_{\infty}(G)$, and is right invariant in the sense that $f x \in \operatorname{RUC}(G)$ whenever $f \in \operatorname{RUC}(G)$.

If $X$ is a right invariant, unital subspace of $l_{\infty}(G)$, then an element $m \in X^{*}$ is called a left invariant mean if $m(1)=1=\|m\|$ and $m(f x)=m(f)$ for all $f \in X, x \in G$. Let $\mathscr{L}(X)$ be the set of left invariant means on $X$.

Received by the editors September 10, 1990.

1980 Mathematics Subject Classification (1985 Revision). Primary 46L05. 
The following simple result is well known but fundamental for theorems of de la Harpe and the present note.

Proposition 1. Let $H$ be a topological group and $G$ be a dense subgroup of $H$ with the relative topology. Then $\mathscr{L}(\operatorname{RUC}(H)) \neq \varnothing$ if and only if $\mathscr{L}(\operatorname{RUC}(G)) \neq$ $\varnothing$.

Proof. By [1, Theorem 2, p. 190], each $f \in \operatorname{RUC}(G)$ extends to a unique function $\bar{f} \in \operatorname{RUC}(H)$. The map $f \rightarrow \bar{f}$ is a linear isometry from $\operatorname{RUC}(G)$ onto $\operatorname{RUC}(H)$, and $\overline{f x}=\bar{f} x$ for $f \in \operatorname{RUC}(G), x \in G$. If $m \in \mathscr{L}(\operatorname{RUC}(H))$, then $f \rightarrow m(\bar{f})$ is in $\mathscr{L}(\operatorname{RUC}(G))$. Conversely, if $n \in \mathscr{L}(\operatorname{RUC}(G))$, then define $\bar{n} \in \operatorname{RUC}(H)^{*}$ by: $\bar{n}(g)=n\left(\left.g\right|_{G}\right)$. Then $\bar{n}$ is a mean, and $\bar{n}(g x)=$ $\bar{n}(g)$ for $x \in G$. Since the map $x \rightarrow g x$ is norm continuous on $H$, it follows that $\bar{n} \in \mathscr{L}(\operatorname{RUC}(H))$.

We need de la Harpe's theorem [4; 5, (2.35)] in the proof of the result of this note. For the sake of completeness, we sketch the proof.

Let $M$ be a von Neumann algebra realized on a Hilbert space $\mathbf{K}$. Let $H$ be the unitary group of $M$ with the ultraweak topology. Now on $H$, the latter topology coincides with both the weak operator and strong operator topologies on $H$. Since the involution is weak operator continuous and multiplication is strong operator continuous on $H$, it follows that $H$ is a topological group. (de la Harpe uses the strong operator topology in [4], but it is important for our purposes that the (intrinsic) ultraweak topology be used.)

Haagerup [3] notes that the separability condition of [4] is not essential.

Theorem 1 [4]. The von Neumann algebra $M$ is injective if $\mathscr{L}(\operatorname{RUC}(H)) \neq \varnothing$.

Proof. Let $M$ be injective. Suppose first that $M$ is countably generated. Then there exists a net $\left\{H_{\delta}\right\}$ of upwards directed, finite-dimensional unitary subgroups of $H$ with $H^{\prime}=\bigcup H_{\delta}$ dense in $H$. Let $n_{\delta}$ be the mean on $H^{\prime}$ given by: $n_{\delta}(f)=m_{\delta}\left(\left.f\right|_{H_{\delta}}\right)$ where $m_{\delta}$ is Haar measure on $H_{\delta}$. Any weak* cluster point of $\left\{n_{\delta}\right\}$ gives an element of $\mathscr{L}\left(\operatorname{RUC}\left(H^{\prime}\right)\right)$. Hence $\mathscr{L}(\operatorname{RUC}(H)) \neq \varnothing$ by the Proposition.

Now remove the countably generated restriction. Then ([2]) $M$ is generated by an upwards directed collection of injective countably generated sub-von Neumann algebras. A similar argument to that above gives $\mathscr{L}(\operatorname{RUC}(H)) \neq \varnothing$.

Conversely, suppose that there exists $m \in \mathscr{L}(\operatorname{RUC}(H))$ and let $T \in B(\mathbf{K})$. One readily checks that for $\xi, \eta \in \mathbf{K}$, the function $f_{\xi, \eta} \in \operatorname{RUC}(H)$, where $f_{\xi, \eta}(U)=U T U^{-1} \xi \cdot \eta$. Define $T^{\prime} \in B(\mathbf{K})$ by:

$$
T^{\prime} \xi \cdot \eta=m\left(f_{\xi, \eta}\right) \text {. }
$$

By approximating $m$ by convex combinations of point masses, we see that $T^{\prime}$ is in the weak operator closure of $\operatorname{co}\left\{U T U^{-1}: U \in H\right\}$. The invariance of $m$ gives $T^{\prime} \in M^{\prime}$. Hence $M$ has Property $\mathrm{P}$ and so is injective.

We now come to our characterization of nuclearity.

Theorem 2. Let $A$ be a unital $C^{*}$-algebra with unitary group $G$, and give $G$ the relative weak topology (as a subset of the Banach space $A$ ). Then $G$ is a topological group, and $A$ is nuclear if and only if there exists a left invariant mean on $\operatorname{RUC}(G)$. 
Proof. Regard $A \subset A^{* *}$. Then $A^{* *}$ is a von Neumann algebra and its unitary group $H$ is a topological group in the ultraweak topology. Further, since the weak topology on $A$ coincides with the relative ultraweak topology, it follows that the topology on $G$ is the relative topology inherited from $H$. Also, $G$ is dense in $H$ [6, (2.3.3)], and $A$ is nuclear if and only if $A^{* *}$ is injective. The results now follows using Theorem 1 and the proposition.

Corollary 1. The following statements are equivalent for a unital $C^{*}$-algebra with unitary group $G$.

(1) $A$ is nuclear.

(2) If $K$ is a nonempty compact, convex subset of a locally convex space and $G$ has an affine left action on $K$, which is jointly continuous, then there is a $G$-fixed point in $K$.

Proof. Use the fixed-point theorem [5, (2.23)].

\section{ACKNOWLEDGMENT}

Most of the work for this note was done while the author was visiting UCLA in the summer of 1990 at the kind ir.vitation of Ed Effros. The author gratefully acknowledges NSF support for that period, and the highly stimulating suggestions of and conversations with Ed Effros and Jerry Kaminker.

\section{REFERENCES}

1. N. Bourbaki, General topology, Addison-Wesley, Reading, MA, 1966.

2. G. A. Elliott, On approximately finite-dimensional von Neumann algebras. II, Canad. Math. Bull 21 (1978), 415-418.

3. U. Haagerup, All nuclear $C^{*}$-algebras are amenable, Invent. Math. 74 (1983), 305-319.

4. P. de la Harpe, Moyennabilité du groupe unitaire et propriété $P$ de Schwartz des algèbres de von Neumann, Lecture Notes in Math., vol. 725, Springer-Verlag, New York, 1979.

5. A. L. T. Paterson, Amenability, Math. Surveys and Monogr., no. 29, Amer. Math. Soc., Providence, RI, 1988.

6. G. K. Pederson, $C^{*}$-algebras and their automorphism groups, Academic Press, London, 1979. 